\title{
Optic Disc Hemorrhage after Phacoemulsification in Patients with Glaucoma
}

\author{
Karine D. Bojikian, Daniel B. Moore, Philip P. Chen, and Mark A. Slabaugh \\ Department of Ophthalmology, University of Washington, Box 359608, 325 Ninth Avenue, Seattle, WA 98104-2499, USA \\ Correspondence should be addressed to Mark A. Slabaugh; mas12@uw.edu
}

Received 30 December 2013; Accepted 27 January 2014; Published 5 March 2014

Academic Editors: B. V. Bui, L. Racette, and I.-J. Wang

Copyright (C) 2014 Karine D. Bojikian et al. This is an open access article distributed under the Creative Commons Attribution License, which permits unrestricted use, distribution, and reproduction in any medium, provided the original work is properly cited.

\begin{abstract}
Background. Optic disk hemorrhage is known to be a risk factor for glaucoma progression. Cataract surgery by phacoemulsification results in large intraocular pressure fluctuations. We aim to investigate whether phacoemulsification is associated with optic disc hemorrhage in patients with glaucoma. Methods. This is a retrospective review of consecutive university clinic based glaucoma patients undergoing phacoemulsification alone, with at least 3 visits in the year before and at least 5 visits in the year following phacoemulsification. The presence of optic disk hemorrhage was evaluated with slit lamp biomicroscopy at each clinic visit prior to and following phacoemulsification. Results. We evaluated 158 eyes of 158 subjects; 15 (9.5\%) had ODH noted at least once during the 2-year study period. Four eyes had ODH identified on postoperative day 1, for a cross-sectional prevalence of $2.5 \%$. Fourteen $\mathrm{ODH}$ episodes were noted preoperatively versus 12 episodes postoperatively $(P=0.68)$. Aspirin use was associated with ODH $(P=0.015)$. Conclusions. Our cross-sectional study found a prevalence of ODH immediately after CE that was similar to other published rates, and our longitudinal study did not find an increase in ODH in the year after phacoemulsification when compared to the year prior to surgery.
\end{abstract}

\section{Introduction}

Glaucoma is characterized by retinal ganglion cell degeneration, characteristic changes of the optic disc, and associated visual field loss. Elevated intraocular pressure (IOP) remains the most important known risk factor for the development and progression of glaucoma $[1,2]$. Optic disc hemorrhage $(\mathrm{ODH})$ has been associated with glaucoma damage and is considered to be an independent risk factor both for development and progression of the disease [3-5].

The etiology of ODH is poorly understood. Some authors have suggested that it represents rupture of anterior capillaries during posterior bowing of the lamina cribrosa, small infarctions in the capillaries of the optic nerve head, or other poorly defined vascular or connective tissue insults [6]. In spite of its unknown cause, multiple cross-sectional, observational, and prospective studies have identified it as an independent risk factor for progression of glaucomatous visual field loss. As a clinical sign, it remains one of the clearest indicators of optic neuropathy when caring for or identifying individuals with glaucoma.
During cataract extraction by phacoemulsification (CE), high absolute IOP and large IOP fluctuations occur intraoperatively and in the early postoperative period $[7,8]$. Although the long-term clinical significance of these shortterm IOP fluctuations in glaucoma patients is not clear, these transient high pressures have been documented to cause increased disc cupping and acute reversible visual field depression $[9,10]$. To determine whether such large but shortterm IOP fluctuations are associated with development of $\mathrm{ODH}$, we examined the cross-sectional prevalence of ODH immediately after $\mathrm{CE}$ and the period prevalence of $\mathrm{ODH}$ in glaucoma patients undergoing $\mathrm{CE}$.

\section{Methods}

We performed a retrospective review of all glaucoma patients undergoing phacoemulsification as a sole procedure, performed by one surgeon between August 1996 and July 2011 at the University of Washington. This study was approved by the Human Subjects Division of the University of Washington. 
TABLE 1: Baseline and clinical characteristics of the population studied $(N=158)$, results given as mean \pm S.D. where applicable.

\begin{tabular}{lc}
\hline Age (years) & $74.4 \pm 9.74$ \\
Sex & (range 64-92) \\
Female & $96(61 \%)$ \\
Male & $62(39 \%)$ \\
Race & \\
Caucasian & $124(78 \%)$ \\
Asian & $18(11 \%)$ \\
African American & $12(8 \%)$ \\
Others & $4(3 \%)$ \\
Glaucoma type & \\
Primary open angle & $87(56 \%)$ \\
Normal tension & $26(16 \%)$ \\
Pseudoexfoliation & $26(16 \%)$ \\
Chronic angle closure & $12(8 \%)$ \\
Others & $7(4 \%)$ \\
Visual field (n= 140) & \\
Mean deviation (dB) & \\
Pattern standard deviation $(\mathrm{dB})$ & $15.1 \pm 2.9$ \\
Preoperative intraocular pressure (mmHg) & $16.7 \pm 3.3$ \\
Preoperative number of medications & $1.9 \pm 0.9$ \\
Postoperative intraocular pressure $(\mathrm{mmHg})$ & \\
Postoperative number of medications & $4.51 \pm 3.70$ \\
\hline
\end{tabular}

The diagnosis of glaucoma was based on characteristic optic nerve findings and/or visual field loss, irrespective of IOP. The indication for cataract surgery in all patients was visual acuity that was felt to be reduced by a visually significant cataract. Inclusion criteria for all patients included at least 3 visits in the year prior to scheduled $\mathrm{CE}$ and at least 5 visits in the year following $\mathrm{CE}$, including at least 3 visits in the perioperative period (defined as the 6 weeks immediately postoperatively). Exclusion criteria included prior trabeculectomy or other incisional glaucoma surgeries. In cases where both eyes of one patient were eligible, the eye undergoing surgery first was chosen for the study.

Pertinent clinical information prior to and after CE was recorded, as well as intraoperative data. This included baseline demographics, diagnosis subtypes, ophthalmic biometry obtained by IOL Master (Carl Zeiss Meditec Inc., Dublin, CA, USA) or ultrasound A-scan (Innovative Imaging Inc., Ellex, Minneapolis, MN, USA), preoperative IOP (defined as the mean of all clinic visits prior to surgery during the followup period), postoperative IOP (defined as the mean of all clinic visits after surgery excluding the six-week perioperative visits), postoperative IOP spike (defined as IOP at least 50\% higher than the preoperative IOP during the immediate postoperative period), number of medications, and disease severity indices (visual field mean deviation and pattern standard deviation).

A single glaucoma subspecialist (PPC) examined and treated each of the patients throughout the study period.
During each clinic visit, the presence or absence of ODH was specifically determined and noted in the chart using slit lamp biomicroscopy, and, if present, the location was recorded. An $\mathrm{ODH}$ was defined as a discrete splinter-shaped area of hemorrhage seen on the optic disc rim, or touching it. Given that ODH has been found to persist for up to 6-8 weeks [8], an $\mathrm{ODH}$ was considered new if it recurred at least 2 months later if in the same location, or if it occurred at a different location from a previous $\mathrm{ODH}$. The period prevalence was defined as the proportion of the total population that showed $\mathrm{ODH}$ over the time period studied. The incidence rate of $\mathrm{ODH}$ was calculated from the number of all detected ODH per patient per year.

Statistical analysis was carried out using PASW Statistics 18.0.0 (IBM SPSS Inc.; New York, NW, USA). Univariate analysis was performed using 2-tailed Student's $t$-test for continuous variables, Pearson chi-square and Fisher's exact test for categorical variables, and Mann-Whitney $U$ test for ordinal variables.

\section{Results}

We included 158 eyes of 158 patients who had CE but reviewed notes from both eyes of all patients for notation of $\mathrm{ODH}$ to determine incidence per patient and to further examine the relationship between $\mathrm{CE}$ and $\mathrm{ODH}$ (Table 1). Four eyes had a new ODH noted on postoperative day 1 (POD1), for a cross-sectional prevalence of $2.5 \%$. In another three eyes, an $\mathrm{ODH}$ was observed at the same location at the clinic visit preceding CE (range, 2-4 weeks prior to surgery) and on POD1. At postoperative month 1, the ODH persisted in one of these eyes, and in two eyes the ODH seen preoperatively had resolved. Three of four ODH initially identified on POD1 remained at postoperative month 1 .

Of the 158 study eyes, fifteen (9.5\%) were recorded as having $\mathrm{ODH}$ at least once during the two-year study period. Eight of 15 study eyes (53\%) had more than one ODH, and a total of 26 new ODH episodes were observed (14 episodes preoperatively versus 12 episodes postoperatively, $P=0.68$ ). Four eyes had ODH noted in both the pre- and postoperative year. Eleven of 15 patients (73\%) with ODH were using aspirin at the time the ODH was identified $(P=0.015)$; no other significant differences were detected between those with and without ODH for any of the factors examined (Table 2).

We compared the period prevalence of $\mathrm{ODH}$ before and after CE (including the eyes with ODH seen on POD 1 as described above). During the one year prior to CE, 9 eyes (5.7\%) had ODH noted, compared with 10 eyes (6.3\%) during the one year after $\mathrm{CE}(P=0.81)$. Notably, significantly more visits occurred during the year after surgery ( $6.4 \pm 1.8$ versus $3.9 \pm 1.2, P<0.001)$. We determined the period prevalence of ODH episodes for each 3-month time period before and after $\mathrm{CE}$ and found no significant differences between the preoperative and postoperative periods $(P=0.37$, Table 3$)$.

As an additional comparison and control group, we determined $\mathrm{ODH}$ incidence per patient and the $\mathrm{ODH}$ period prevalence from the nonsurgical eye. Ten patients $(6.3 \%)$ had $\mathrm{ODH}$ only in the study eye, five patients (3.1\%) had bilateral 
TABLE 2: Selected risk factors evaluated between patients who did and did not have an optic disc hemorrhage.

\begin{tabular}{|c|c|c|c|}
\hline Risk factor & $\mathrm{ODH}(n=15,9 \%)$ & No ODH $(n=143,91 \%)$ & $P$ \\
\hline Age (years) & $76.0 \pm 7.5$ & $74.2 \pm 9.9$ & 0.499 \\
\hline Sex (male) & $3(20 \%)$ & $59(41 \%)$ & $0.109^{9}$ \\
\hline Race & & & $0.106^{\dagger}$ \\
\hline Caucasian & $14(93 \%)$ & $110(77 \%)$ & \\
\hline Asian & 0 & $18(13 \%)$ & \\
\hline African American & 0 & $12(8 \%)$ & \\
\hline Others & $1(7 \%)$ & $3(2 \%)$ & \\
\hline Axial length (mm) & $23.79 \pm 1.88$ & $24.41 \pm 1.95$ & $0.243^{\S}$ \\
\hline Glaucoma type & & & $0.358^{\dagger}$ \\
\hline Primary open angle & $7(47 \%)$ & $80(56 \%)$ & \\
\hline Pseudoexfoliation & $5(33 \%)$ & $21(15 \%)$ & \\
\hline Normal tension & $1(7 \%)$ & $25(17 \%)$ & \\
\hline Chronic angle closure & $2(13 \%)$ & $10(7 \%)$ & \\
\hline Others & 0 & $7(5 \%)$ & \\
\hline Preoperative visits & $3.9 \pm 1.3$ & $3.6 \pm 0.8$ & $0.454^{\S}$ \\
\hline Postoperative visits & $9.1 \pm 2.6$ & $9.9 \pm 2.1$ & $0.266^{\S}$ \\
\hline Preoperative IOP $(\mathrm{mmHg})$ & $15.9 \pm 3.1$ & $16.7 \pm 3.3$ & $0.332^{\S}$ \\
\hline Postoperative IOP (mmHg) & $14.2 \pm 2.4$ & $15.2 \pm 2.9$ & $0.102^{\S}$ \\
\hline IOP spike & $2(13.3 \%)$ & $23(16 \%)$ & $0.808^{\dagger}$ \\
\hline Preoperative medications & $2.13 \pm 1.25$ & $1.92 \pm 0.93$ & $0.407^{\ddagger}$ \\
\hline Postoperative medications & $2.13 \pm 1.25$ & $1.92 \pm 1.1$ & $0.479^{\ddagger}$ \\
\hline Visual field $(\mathrm{dB})(n=140)$ & $(n=14)$ & $(n=125)$ & \\
\hline Mean deviation & $-6.39 \pm 4.56$ & $-5.73 \pm 6.03$ & $0.692^{\S}$ \\
\hline Pattern standard deviation & $5.18 \pm 4.44$ & $4.43 \pm 3.62$ & $0.474^{\S}$ \\
\hline CCT (microns) $(n=113)$ & $538 \pm 25$ & $534 \pm 40$ & $0.790^{\S}$ \\
\hline Aspirin use & $11(73 \%)$ & $58(40 \%)$ & $0.015^{\dagger}$ \\
\hline Hypertension & $10(67 \%)$ & $102(71 \%)$ & $0.705^{\dagger}$ \\
\hline Diabetes & $2(13 \%)$ & $32(22 \%)$ & $0.417^{\dagger}$ \\
\hline
\end{tabular}

ODH: optic disc hemorrhage; IOP: intraocular pressure; CCT: central corneal thickness.

${ }^{\dagger}$ Pearson chi-square, ${ }^{\ddagger}$ Mann Whitney $U$ test, ${ }^{{ }^{S}} 2$-tail independent sample $t$-test, and ${ }^{\boldsymbol{g}}$ Fisher's exact test.

TABLE 3: Period prevalence of optic disc hemorrhage episodes for each 3-month time period before and after cataract extraction by phacoemulsification.

\begin{tabular}{lccc}
\hline Time period & Preoperative period & Postoperative period & $P$ \\
\hline $0-3$ months & $3.2 \%$ & $3.8 \%$ & \\
$4-6$ months & $3.2 \%$ & $1.3 \%$ & 0.377 \\
7-9 months & $0.6 \%$ & $1.9 \%$ & \\
$10-12$ months & $1.9 \%$ & $0.6 \%$ & \\
\hline
\end{tabular}

$\mathrm{ODH}$, and two patients $(1.2 \%)$ had ODH only in the fellow eye (period prevalence of $10.7 \%$ in 2 years); the between-eyes correlation of $\mathrm{ODH}$ was significant $(R=.455, P<0.001$, Pearson).

\section{Discussion}

Although the precise cause of ODH is still unknown, its prognostic importance for the progression of glaucoma has been well established. The Collaborative Normal Tension
Glaucoma Study showed higher rates of progression in glaucoma patients with $\mathrm{ODH}$ at baseline, and the Early Manifest Glaucoma Trial found ODH seen during followup to be an independent predictor of glaucoma progression, the risk increasing along with increasing ODH frequency $[3,4]$. Another clinic-based cohort of 348 glaucoma patients followed for an average of 8.2 years reported twice the rate of visual field progression in eyes that had an $\mathrm{ODH}$ at any time during follow-up [5].

Phacoemulsification is performed with a relatively closed infusion and aspiration system, resulting in repeated transiently high IOP and large IOP fluctuations during surgery. Depending on the irrigation fluid bottle height, vacuum, and aspiration settings, IOP frequently exceeds $70 \mathrm{mmHg}$; however, at times the effective IOP may precipitously drop close to $0 \mathrm{mmHg}$ [7]. During the first 24 hours after CE, up to $40 \%$ of glaucoma patients can have a significant increase in IOP [11]. Animal models suggest that this level of IOP fluctuation may cause significant acute laminar deformation in eyes with early to moderate glaucomatous damage [12]. 
Several studies have attempted to discover factors associated with $\mathrm{ODH}$ in glaucoma patients and although some have suggested associations between $\mathrm{ODH}$ and hypertension diabetes mellitus, increasing IOP, pseudoexfoliation, and use of aspirin as significant, others could not confirm these findings [13-20]. In the present study, hypertension and diabetes were not associated with $\mathrm{ODH}$, but the use of aspirin was associated with ODH. Given its inhibitory effect on platelet aggregation, aspirin could either increase the risk of developing $\mathrm{ODH}$, could lead to larger $\mathrm{ODH}$ that are more likely to be detected, or both. Another possible explanation is that patients using aspirin are more likely to have vascular diseases that may predispose to the development of ODH. The high intereye correlation for $\mathrm{ODH}$ that we found is also consistent with the intereye correlation that is known to be present in the progression in glaucoma patients [21].

This study is limited by its retrospective nature. We performed only clinical examination for ODH presence, and this could have led to an underestimation of ODH prevalence. However, presence or absence of ODH was specifically noted for every patient visit and patients were excluded from the study when the optic disc could not be adequately visualized. Also, comparison of studies using solely clinical examination to detect ODH (2.55 to $8.16 \%$ /year) versus photography (1.41 to $14.10 \%$ /year) versus both modalities ( 0.22 to $6.88 \%$ /year) shows that the period prevalence reported in the literature is similar using different methods [3, 5, 22-25]. Our crosssectional prevalence rate of new $\mathrm{ODH}$ on postoperative day 1 (POD1) was $2.5 \%$, which is comparable with that reported in other cross-sectional studies $(2 \%$ to $14.1 \%)[3,6,13,14]$. Our period prevalence rate of $10.7 \%$ in 2 years (5.35\%/year) is also comparable with that reported in other prospective $(0.22 \%$ to $6.88 \%$ /year) and retrospective studies ( $0.67 \%$ to $8.16 \% /$ year) of ODH $[5,6,15-20]$.

Notably, the additional clinic visits associated with postoperative care resulted in a significantly higher number of clinic visits during the postoperative year compared to the preoperative year. This should result in increased surveillance for ODH and would be expected to bias our findings towards greater detection of $\mathrm{ODH}$ during the postoperative year compared to the preoperative year, yet we did not detect any difference from the preoperative visits.

In conclusion, our results imply that the supraphysiologic short-term IOP fluctuation experienced during phacoemulsification does not commonly lead to an increased incidence of $\mathrm{ODH}$ in eyes with glaucoma. We hypothesize that disk hemorrhages are more likely to be a result of either vascular events that are not affected by large swings in IOP or are a result of smaller but more chronic changes in lamina cribrosa position.

\section{Conflict of Interests}

No author has any financial conflict of interests.

\section{Acknowledgment}

This study was funded in part by an unrestricted departmental grant from Research to Prevent Blindness.

\section{References}

[1] M. C. Leske, A. M. S. Connell, S.-Y. Wu, L. G. Hyman, and A. P. Schachat, "Risk factors for open-angle glaucoma: the Barbados eye study," Archives of Ophthalmology, vol. 113, no. 7, pp. 918924, 1995.

[2] A. Sommer, J. M. Tielsch, J. Katz et al., "Relationship between intraocular pressure and primary open angle glaucoma among white and black Americans: the Baltimore eye survey," Archives of Ophthalmology, vol. 109, no. 8, pp. 1090-1095, 1991.

[3] S. Drange, D. R. Anderson, and M. Schulzer, "Risk factors for progression of visual field abnormalities in normal-tension glaucoma," American Journal of Ophthalmology, vol. 131, no. 6, pp. 699-708, 2001.

[4] M. C. Leske, A. Heijl, M. Hussein, B. Bengtsson, L. Hyman, and E. Komaroff, "Factors for glaucoma progression and the effect of treatment: the early manifest glaucoma trial," Archives of Ophthalmology, vol. 121, no. 1, pp. 48-56, 2003.

[5] F. A. Medeiros, L. M. Alencar, P. A. Sample, L. M. Zangwill, R. Susanna Jr., and R. N. Weinreb, "The relationship between intraocular pressure reduction and rates of progressive visual field loss in eyes with optic disc hemorrhage," Ophthalmology, vol. 117, no. 11, pp. 2061-2066, 2010.

[6] T. A. Uhler and J. Piltz-Seymour, "Optic disc hemorrhages in glaucoma and ocular hypertension: implications and recommendations," Current Opinion in Ophthalmology, vol. 19, no. 2, pp. 89-94, 2008.

[7] H. R. Wilbrandt and T. H. Wilbrandt, "Evaluation of intraocular pressure fluctuations with differing phacoemulsification approaches," Journal of Cataract and Refractive Surgery, vol. 19, no. 2, pp. 223-231, 1993.

[8] H. Levkovitch-Verbin, Z. Habot-Wilner, N. Burla et al., "Intraocular pressure elevation within the first 24 hours after cataract surgery in patients with glaucoma or exfoliation syndrome," Ophthalmology, vol. 115, no. 1, pp. 104-108, 2008.

[9] A. Azuara-Blanco, A. Harris, L. B. Cantor, M. M. Abreu, and M. Weinland, "Effects of short term increase of intraocular pressure on optic disc cupping," British Journal of Ophthalmology, vol. 82, no. 8, pp. 880-883, 1998.

[10] J. R. Trible and D. R. Anderson, "Factors associated with intraocular pressure-induced acute visual field depression," Archives of Ophthalmology, vol. 115, no. 12, pp. 1523-1527, 1997.

[11] H. Yasutani, K. Hayashi, H. Hayashi, and F. Hayashi, "Intraocular pressure rise after phacoemulsification surgery in glaucoma patients," Journal of Cataract and Refractive Surgery, vol. 30, no. 6, pp. 1219-1224, 2004.

[12] H. Yang, H. Thompson, M. D. Roberts, I. A. Sigal, J. C. Downs, and C. F. Burgoyne, "Deformation of the early glaucomatous monkey optic nerve head connective tissue after acute IOP elevation in 3-D histomorphometric reconstructions," Investigative Ophthalmology and Visual Science, vol. 52, no. 1, pp. 345-363, 2011.

[13] B. Klein, R. Klein, W. E. Sponsel et al., "Prevalence of glaucoma: the Beaver Dam eye study," Ophthalmology, vol. 99, no. 10, pp. 1499-1504, 1992.

[14] P. R. Healey, P. Mitchell, W. Smith, and J. J. W. Jie Jin Wang, "Optic disc hemorrhages in a population with and without signs of glaucoma," Ophthalmology, vol. 105, no. 2, pp. 216-223, 1998.

[15] M. T. Rasker, A. van den Enden, D. Bakker, and P. F. Hoyng, "Deterioration of visual fields in patients with glaucoma with and without optic disc hemorrhages," Archives of Ophthalmology, vol. 115, no. 10, pp. 1257-1262, 1997. 
[16] A. S. Soares, P. H. Artes, P. Andreou, R. P. Leblanc, B. C. Chauhan, and M. T. Nicolela, "Factors associated with optic disc hemorrhages in glaucoma," Ophthalmology, vol. 111, no. 9, pp. 1653-1657, 2004.

[17] D. L. Diehl, H. A. Quigley, N. R. Miller, A. Sommer, and E. N. Burney, "Prevalence and significance of optic disc hemorrhage in a longitudinal study of glaucoma," Archives of Ophthalmology, vol. 108, no. 4, pp. 545-550, 1990.

[18] K. Grødum, A. Heijl, and B. Bengtsson, "Optic disc hemorrhages and generalized vascular disease," Journal of Glaucoma, vol. 11, no. 3, pp. 226-230, 2002.

[19] A. Tuulonen, T. Takamoto, D.-C. Wu, and B. Schwartz, "Optic disk cupping and pallor measurements of patients with a disk hemorrhage," American Journal of Ophthalmology, vol. 103, no. 4, pp. 505-511, 1987.

[20] B. Bengtsson, M. C. Leske, Z. Yang, and A. Heijl, "Disc hemorrhages and treatment in the early manifest glaucoma trial," Ophthalmology, vol. 115, no. 11, pp. 2044-2048, 2008.

[21] P. P. Chen, "Correlation of visual field progression between eyes in patients with open-angle glaucoma," Ophthalmology, vol. 109, no. 11, pp. 2093-2099, 2002.

[22] J. W. Hsieh, Y. W. Lan, I. J. Wang, and F. J. Sun, "Clinical characteristics and prognostic significance of disc hemorrhage in open-angle and angle-closure glaucoma," Journal of Glaucoma, vol. 19, no. 7, pp. 483-487, 2010.

[23] K. Ishida, T. Yamamoto, K. Sugiyama, and Y. Kitazawa, "Disk hemorrhage is a significantly negative prognostic factor in normal-tension glaucoma," American Journal of Ophthalmology, vol. 129, no. 6, pp. 707-714, 2000.

[24] M. H. Suh and K. H. Park, "Period prevalence and incidence of optic disc haemorrhage in normal tension glaucoma and primary open-angle glaucoma," Clinical and Experimental Ophthalmology, vol. 39, no. 6, pp. 513-519, 2011.

[25] C. G. V. De Moraes, V. J. Juthani, J. M. Liebmann et al., "Risk factors for visual field progression in treated glaucoma," Archives of Ophthalmology, vol. 129, no. 5, pp. 562-568, 2011. 


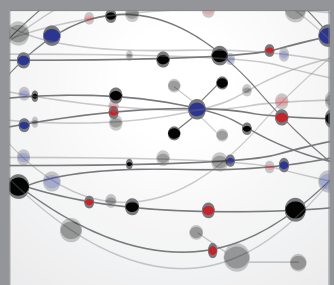

The Scientific World Journal
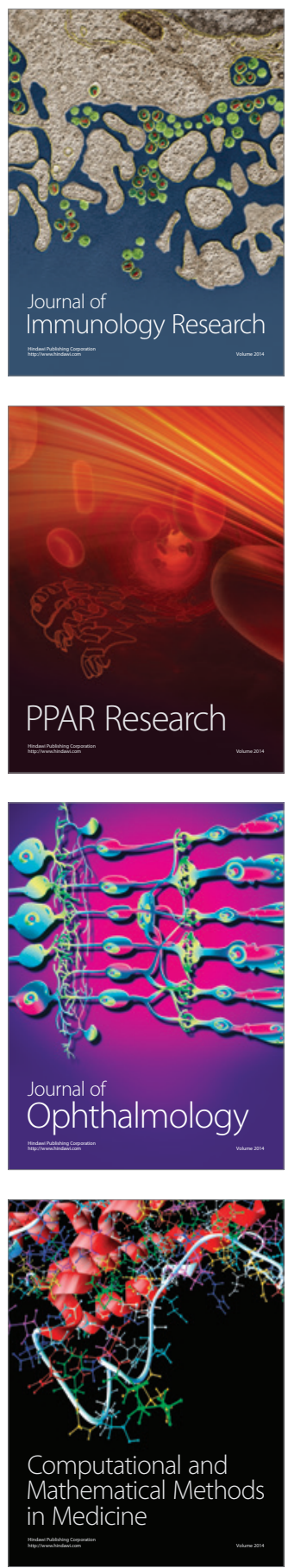

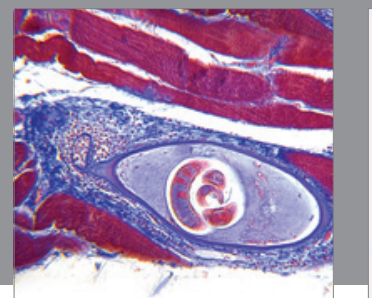

Gastroenterology

Research and Practice
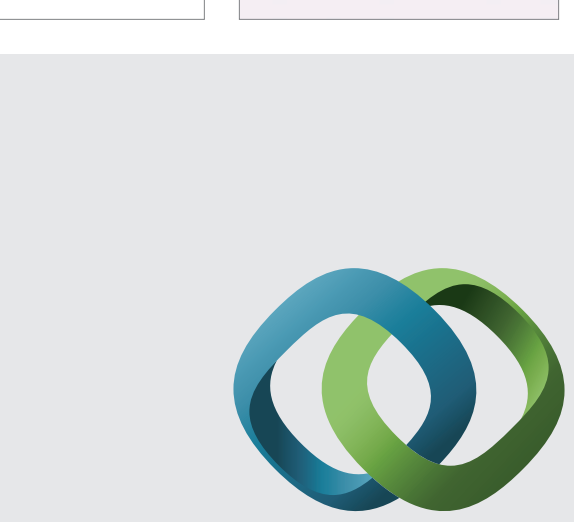

\section{Hindawi}

Submit your manuscripts at

http://www.hindawi.com
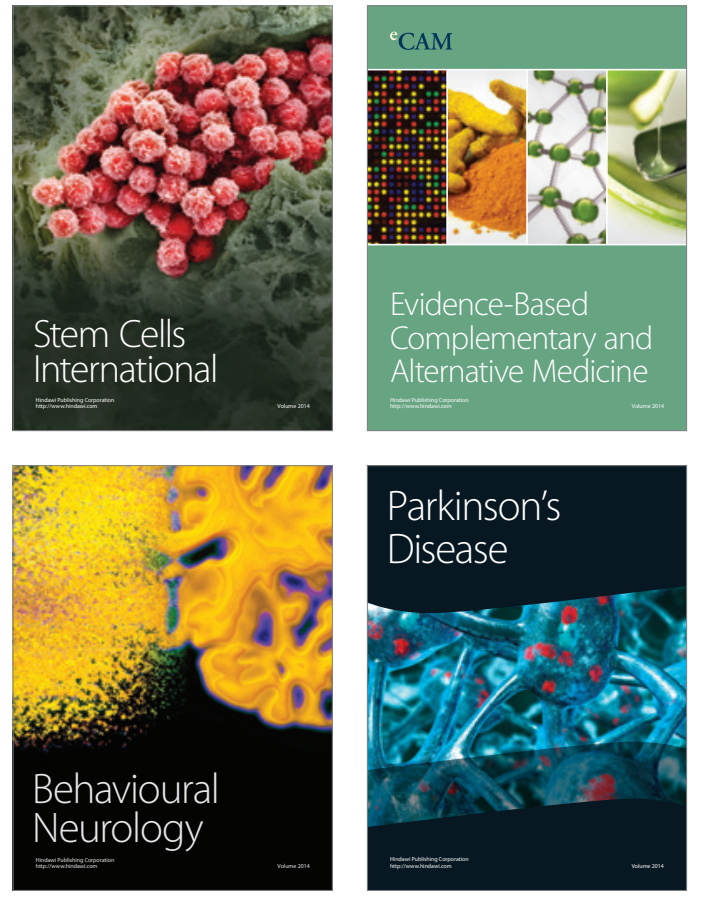
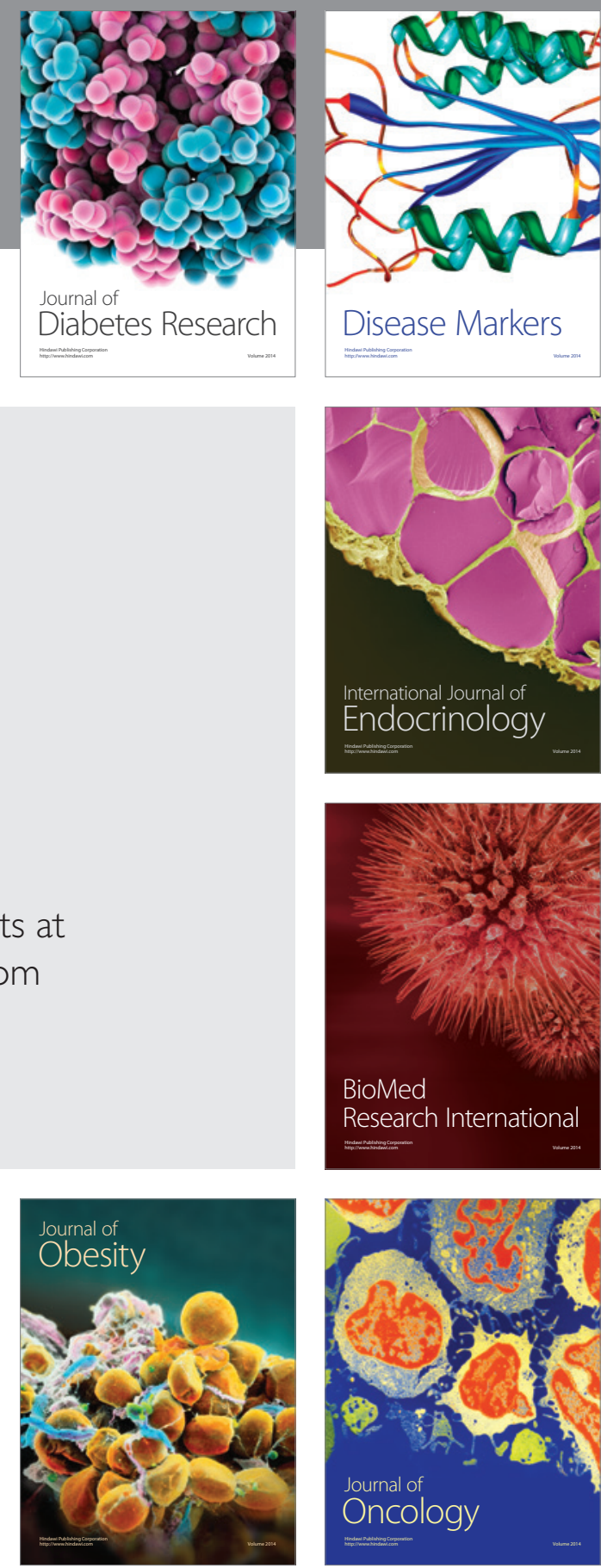

Disease Markers
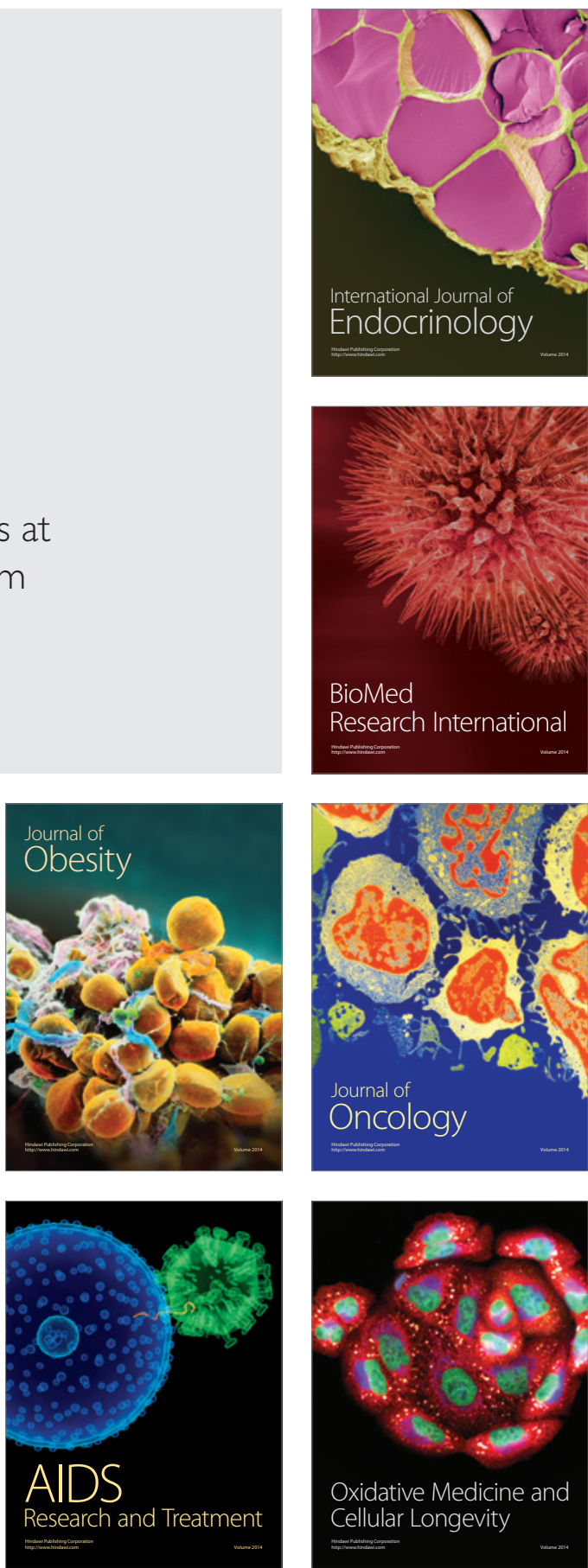